\title{
ON THE CONNECTION BETWEEN GAPS IN POWER SERIES AND THE ROOTS OF THEIR PARTIAL SUMS
}

\author{
BY \\ P. ERDOSS AND H. FRIED( $($ )
}

In this paper we are going to investigate the connections between the gaps of power series with the distribution of the roots of their partial sums. Let

$$
f(x)=1+a_{1} x+\cdots+a_{n} x^{n}+\cdots
$$

be a power series with the radius of convergence 1 . We say that it has Ostrowski gaps $\rho$ if there exists a $\rho<1$ and a pair of infinite sequences $m_{k}$ and $n_{k}$, with $m_{k}<n_{k}$ and $\lim n_{k} / m_{k}>1$, such that $\left|a_{n}\right|<\rho^{n}$ for $m_{k} \leqq n \leqq n_{k}$.

It has infinite Ostrowski gaps $\rho(\rho<1)$ if to every $\rho^{\prime}>\rho$ there corresponds a pair of infinite sequences $m_{k}$ and $n_{k}$ (depending on $\rho^{\prime}$ ) with $m_{k}<n_{k}$ and $\lim n_{k} / m_{k}=\infty$ such that $\left|a_{n}\right|<\rho^{\prime n}$ for $m_{k} \leqq n \leqq n_{k}$.

We denote by $A(n, r)$ the number of roots of $f(x)=1+a_{1} x+\cdots+a_{n} x^{n}$ within the circle of radius $r$.

It is well known that every overconvergent power series has Ostrowski gaps, and that every power series with Ostrowski gaps is overconvergent in a domain of which every regular point of the circle of convergence is an interior point.

We are going to prove the following theorems:

THEOREM I. A necessary and sufficient condition that a power series have Ostrowski gaps is that there exist an $r>1$, such that

$$
\liminf _{n=\infty} \frac{A(n, r)}{n}<1
$$

THEOREM II. A necessary and sufficient condition that a power series have infinite Ostrowski gaps $\rho$ is that

$$
\liminf _{n=\infty} \frac{A(n, r)}{n}=0 \quad \text { for all } r<\frac{1}{\rho} .
$$

Theorem $I$ is not new. It has been proved by Bourion $\left({ }^{2}\right)$, but his proof is quite different from ours. The proof of Theorem I will be based on the following lemma, which seems interesting in itself.

Presented to the Society, September 12, 1943; received by the editors September 25, 1946.

(1) Deceased December 23, 1945.

() L'ultra convergence dans les series de Taylor, Actualités Scientifique et Industriel, no. 472, Paris, 1937. 
Lemma I. If $0<\rho<1$ and $1 / \rho>r>1$, then there exists a constant $c>0$ (depending only on $r$ and $\rho$ ) such that every equation $f_{n}(x)=1+a_{1} x+\cdots+a_{n} x^{n}$ $=0$, in which

$$
\left|a_{k}\right|<\rho^{k} \quad(m \leqq k \leqq n),
$$

has at least $c(n-m+1)$ roots outside the circle of radius $r$.

Proof. Without loss of generality we can assume $m>n / 2$. Since the product of the moduli of the roots of our equation is $\left|1 / a_{n}\right| \geqq \rho^{-n}$, at least one of the roots exceeds $r$. Therefore $N /(n-m+1)>0$, where $N$ denotes the number of roots outside the circle of radius $r$. If the lemma were false there would exist a sequence of polynomials

$$
f_{\nu}(x)=1+a_{1} x+\cdots+a_{m} x^{m}+\cdots+a_{n} x^{n} \quad\left(m=m_{\nu}, n=n_{\nu}\right)
$$

(here and in the future we shall omit the index $\nu$ where there is no danger of confusion) in which $\left|a_{k}\right|<\rho^{k}$, for $m \leqq k \leqq n$, and such that

$$
c=N /(n-m+1) \rightarrow 0
$$

$\left(c=c_{\nu}, N=N_{\nu}\right.$, and so on, $\left.\nu \rightarrow \infty\right)$.

We are going to show that these assumptions lead to a contradiction. We choose

$$
k>\max \left(\frac{1+r}{1-\rho r}, \frac{1}{\rho}\right) .
$$

We write the polynomials (5) in the following form

$$
f_{\nu}(x)=a_{n} \prod_{i}\left(x-y_{i}\right) \prod_{i}\left(x-z_{i}\right) \prod_{i}\left(x-u_{i}\right)=a_{n} Y(x) Z(x) U(x)
$$

where $y_{i}$ denotes the roots for which $\left|y_{i}\right| \leqq r, z_{i}$ the roots for which

$$
r<z_{i} \leqq 2 D, \quad D=k^{n /(n-m+1)},
$$

and $u_{i}$ the roots for which $2 D<u_{i}$. Further we denote by $l, s, t$ the number of roots $y_{i}, z_{i}, u_{i}$ respectively. From (6) we have

$$
\begin{aligned}
\lim \frac{s+t}{n-m+1} & =0 ; \text { hence } \\
\lim \frac{s}{n-m+1} & =\lim \frac{t}{n-m+1}=0, \quad \lim \frac{l}{n}=1 ; \\
\lim \frac{l+s-m+1}{n-m+1} & =\lim \left(1-\frac{t}{n-m+1}\right)=1 ; \\
\lim \frac{l+s-n}{n-m+1} & =\lim \left(-\frac{t}{n-m+1}\right)=0 .
\end{aligned}
$$


From the definition of the $z$ 's it follows that

$$
r^{s}<|Z(0)| \leqq 2^{s} D^{s}
$$

or

$$
r^{s / n}<|Z(0)|^{1 / n} \leqq 2^{s / n} D^{s / n}
$$

Hence from (9)

$$
\lim |Z(0)|^{1 / n}=1 \quad(\nu \rightarrow \infty) .
$$

From

$$
1=\left|a_{n} \cdot Y(0) \cdot Z(0) U(0)\right|
$$

and (10) it follows that

$$
\lim \left|a_{n} Y(0) U(0)\right|^{1 / n}=1 .
$$

If $x$ is any point within the circle of radius $D$ we obtain from the definition of the $u_{i}$ 's that

$$
1 / 2<\left|\left(u_{i}-x\right) / u_{i}\right|<3 / 2
$$

or

$$
(1 / 2)^{t}<\left|U(x) \cdot(U(0))^{-1}\right|<\cdot(3 / 2)^{t} .
$$

Hence from (9)

$$
\lim \left(\left|U(x) \cdot(U(0))^{-1}\right|\right)^{1 / n}=1 \quad\left(U(x)=U_{\nu}(x), n=n_{\nu}\right) .
$$

Let now $\xi$ be the point on the circle of radius $D$ where the product $|Y(x) Z(x)|$ assumes its maximum. It follows from Cauchy's formula that this maximum is greater than $D^{l+8}$. We obtain from

$$
\left|f_{v}(\xi)\right|=\left|a_{n} \cdot Y(\xi) \cdot Z(\xi) \cdot U(\xi)\right| \geqq\left|a_{n} U(\xi)\right| D^{l+8}
$$

and from (11) and (12) that

$$
\left|f_{\nu}(\xi)\right| \geqq D^{l+s}(1-\epsilon)^{n}|Y(0)|^{-1},
$$

for all sufficiently large $\nu$, where $\epsilon$ is an arbitrarily small positive number.

Now we shall show that this is impossible, namely that the maximum of $\left|f_{\nu}(x)\right|$ on the circle of radius $D$ is not as large as that.

Put

$$
\max _{k \leqq n}\left|a_{k}\right|=B_{\nu}
$$

The index of the largest coefficient is clearly less than $m$ (since $\rho<1$ ). Now we estimate $B_{\nu}$. Let $\omega$ be the point on the unit circle where $\left|f_{\nu}(x)\right|$ assumes its maximum. It follows from Cauchy's formula that 


$$
B_{\nu} \leqq\left|f_{\nu}(\omega)\right| \text {. }
$$

From (11) and (12) it follows that

$$
\lim \left|a_{n} Y(0) \cdot U(\omega)\right|^{1 / n}=1 .
$$

(Observe that $k>1$ so that $\omega$ is in the interior of the circle of radius $D$. .) From the definition of the $z_{i}$ we have

$$
r-1 \leqq|z-\omega| \leqq 2 D+1<3 D,
$$

or

$$
(r-1)^{\circ} \leqq|Z(\omega)|<(3 D)^{\circ}
$$

Hence from (9)

$$
\lim (Z(\omega))^{1 / n}=1 .
$$

From $\left|f_{\nu}(\omega)\right|=\left|a_{n} \cdot Y(\omega) \cdot Z(\omega) \cdot U(\omega)\right|$ we obtain by (16) and (15) that

$$
\left|f_{v}(\omega)\right| \leqq(1+r)^{l}(1+\epsilon)^{n} / Y(0) \quad\left(l=l_{v}, \text { and so on }\right)
$$

for all sufficiently large $v$, where $\epsilon$ is an arbitrarily small positive number. From (14) and (17) it follows that

$$
B_{v} \leqq(1+r)^{l}(1+\epsilon)^{n} / Y(0) .
$$

If we denote by $M_{v}$, the maximum of $\left|f_{v}(x)\right|$ on the circle of radius $D$, we have

$$
M, \leqq m_{\nu} \frac{(1+r)^{l}(1+\epsilon)^{n}}{Y(0)} D^{m-1}+\sum_{i=m}^{n}(\rho \cdot D)^{i}
$$

or, because of $\rho D>1$,

$$
M,<m \frac{(1+r)^{l}(1+\epsilon)^{n}}{|Y(0)|} D^{m-1}+(n-m+1)(\rho \cdot D)^{n} .
$$

From (13) and (18) it follows that

$$
\frac{D^{l+\varepsilon}}{|Y(0)|}(1-\epsilon)^{n} \leqq m \frac{(1+r)^{l}(1+\epsilon)^{n}}{|Y(0)|} D^{m-1}+(n-m+1) \rho^{n} D^{n}
$$

for sufficiently large $\nu$ and arbitrarily small positive $\epsilon$. Hence we obtain from $\left|y_{i}\right| \leqq r,(9)$, the definition of $D, m>n / 2$, and (7)

$$
\begin{aligned}
1 & \leqq\left[\frac{m(1+r)^{l}(1+\epsilon)^{n}}{D^{l+\varepsilon-m+1}(1-\epsilon)^{n}}+\frac{(n-m+1) \rho^{n} \cdot \varphi^{l}}{D^{l+s-n}(1-\epsilon)^{n}}\right]^{1 / n} \\
& <\frac{m^{1 / n}(1+r)^{l / n}(1+\epsilon)}{D^{(l+\varepsilon-m+1) / n}(1-\epsilon)}+\frac{(n-m+1)^{1 / n} \rho r^{l / n}}{D^{(l+\varepsilon-n) / n}(1-\epsilon)}<\frac{1+r}{k}+\rho r+\eta<1
\end{aligned}
$$


for every $\eta$ if $\epsilon$ is sufficiently small and $\nu$ sufficiently large. This contradiction establishes the lemma.

Proof of Theorem I. First we show that (2) is necessary. If the power series has Ostrowski gaps there exists a $\rho<1$ and a pair of infinite sequences $m_{k}$ and $n_{k}$ with $m_{k}<n_{k}$ and $\lim n_{k} / m_{k}=\theta(\theta>1)$ such that $\left|a_{n}\right|<\rho^{n}$ for $m_{k} \leqq n \leqq n_{k}$. By Lemma I, corresponding to any $1<r<1 / \rho$ there exists a positive constant $c$ such that

$$
n_{k}-A\left(n_{k}, r\right)>c\left(n_{k}-m_{k}+1\right) .
$$

Hence for sufficiently large $k$

$$
n_{k}-A\left(n_{k}, r\right)>c n_{k}(1-1 / \theta)
$$

or

$$
\frac{n_{k}-A\left(n_{k}, r\right)}{n_{k}}>c\left(1-\frac{1}{\theta}\right)
$$

and therefore

$$
\lim \inf \frac{A(n, r)}{n}<1,
$$

which shows the necessity of condition (2).

Assume now that (2) is satisfied. Then there exists a sequence $n_{k}$ such that

$$
\lim _{k=\infty} \frac{A\left(n_{k}, r\right)}{n_{k}}<1
$$

We denote by $f_{n_{k}}(x)$ the polynomial consisting of the first $n_{k}+1$ terms of $f(x)$, and by $x_{i}^{\left(n_{k}\right)}$ its roots. (To simplify notations we shall omit the index $k$ where there is no danger of confusion.) We choose $\epsilon$ so that $0<\epsilon<r-1$. It is well known that for any $\gamma>0$, only a bounded number of roots of $f_{n_{k}}(x)$, $k=1,2, \cdots$, are within the circle of radius $1-\gamma$. It follows easily from (20) that positive numbers $c$ and $c^{\prime}$ exist, both less than 1 and such that

$$
\left|\Pi^{\prime} x_{i}^{(n)}\right|>(r-\epsilon)^{c n} \quad\left(n=n_{k}\right)
$$

for sufficiently large $k$, where $\left|\Pi^{\prime} x_{i}^{(n)}\right|$ is the product of at least $c^{\prime} n_{k}$ roots of $f_{n_{k}}(x)$. Thus we obtain

$$
a_{n_{k}}<(r-\epsilon)^{-c n k} .
$$

Hence if we choose $\delta$ such that $(r-\epsilon)^{-c}<\rho<1$, we can conclude that $\left|a_{n_{k}}\right|<\rho^{n_{k}}$. Now we choose $\delta$ such that

$$
0<\delta<\rho(r-\epsilon)^{c}-1 .
$$


By Stirling's formula it is easy to see that $C_{n, l n}<(1+\delta)^{n}$ for sufficiently small $l$. Now for

$$
1 \leqq p \leqq l n \text { and } p<\left(1-c^{\prime}\right) n \quad\left(p=p_{k}, n=n_{k}\right)
$$

we obtain

$$
\left|a_{n-p}\right| \leqq C_{n, p}\left|\prod_{i=1}^{p} \xi_{i}\right| /\left|\prod_{i=1}^{n} x_{i}^{(n)}\right|
$$

where $\xi_{1}^{(n)}, \ldots, \xi_{p}^{(n)}$ are the roots with the greatest absolute values. Therefore we have

$$
\left|a_{n-p}\right|<\left(\frac{1+\delta}{(r-\epsilon)^{c}}\right)^{n}<\rho^{n}<\rho^{n-p}
$$

which completes the proof of Theorem I.

For the proof of Theorem II we need the following lemma:

Lemma II. Let $f(z)=1+a_{1} z+\cdots+a_{n} z^{n}+\cdots$ be a power series with Ostrowski gaps $\rho$ and radius of convergence 1 , and let $\epsilon>0$; then for each

$$
r<\left(\frac{1}{\rho}\right)^{\lambda} \text { where } \lambda=\frac{\epsilon}{\sigma+\epsilon} \text { with } \mu=\lim \inf \frac{m_{k}}{n_{k}}
$$

we have

$$
\liminf _{k=\infty} \frac{A\left(n_{k}, r\right)}{n_{k}} \leqq \liminf _{k=\infty} \frac{m_{k}}{n_{k}}+\epsilon
$$

If this lemma were false there would exist an

$$
r_{1}<(1 / \rho)^{\lambda}
$$

such that

$$
\lim _{k=\infty} \frac{A\left(n_{k}, r_{1}\right)}{n_{k}}>\lim _{k=\infty} \frac{m_{k}}{n_{k}}+\epsilon .
$$
that

(We consider if necessary a subsequence of $m_{k}$ and $n_{k}$.) We choose $r_{2}$ so

$$
1<r_{2}<1 / \rho \text { and } r_{1}<\stackrel{\lambda}{r_{2}} .
$$

Thus $r_{1}<r_{2}$. Denote by $M_{n_{k}}(r)$ the maximum of $f_{n_{k}}(x)$ on the circle of radius $r$. From Jensen's formula we have

$$
M_{n}\left(r_{2}\right) \geqq \frac{r_{2}^{\mu_{2}}}{\left|a_{1} \cdot a_{2} \cdots a_{\mu_{2}}\right|}, \quad \quad \mu_{2}=A\left(n, r_{2}\right), \quad n=n_{k},
$$


where $a_{1}, \cdots, a_{\mu_{2}}$ are the roots of $f_{n}(x)$ within the circle of radius $r_{2}$. Hence

$$
M_{n}\left(r_{2}\right) \geqq \frac{r_{2}^{\mu_{2}}}{r_{1}^{\mu_{1}} r_{2}^{\mu_{2}-\mu_{1}}}=\left(\frac{r_{2}}{r_{1}}\right)^{\mu_{1}}, \quad \quad \mu_{1}=A(n, r) .
$$

Since $\left|a_{t}\right|<\rho^{\imath}$ for $m<t<n$ we obtain

$$
M_{n}\left(r_{2}\right) \leqq(1+\eta)^{m} \cdot m \cdot r_{2}^{m}+(n-m+1)\left(\rho r_{2}\right)^{m}
$$

where $\eta$ is arbitrarily small. From (27) and (24) we obtain

$$
\left(M_{n}\left(r_{2}\right)\right)^{1 / n} \geqq\left(\frac{r_{2}}{r_{1}}\right)^{\mu_{1} / n} \geqq\left(\frac{r_{2}}{r_{1}}\right)^{\sigma+\epsilon}
$$

and from (28)

$$
\left(M_{n}\left(r_{2}\right)\right)^{1 / n} \leqq r_{2}^{\sigma}
$$

for sufficiently large $k$. Hence

$$
\left(\frac{r_{2}}{r_{1}}\right)^{\sigma+\epsilon} / r_{2}^{\sigma}=\frac{r_{2}^{\epsilon}}{r_{1}^{\sigma+\epsilon}} \leqq 1
$$

or

$$
r_{2}^{\epsilon /(\sigma+\epsilon)} \leqq r_{1}
$$

which contradicts (25). Thus Lemma II is proved:

Proof of Theorem II. Condition (3) is necessary. This follows immediately from Lemma II; here we have $\lim m_{k} / n_{k}=0$.

Condition (3) is sufficient. If a power series has no infinite Ostrowski gaps $\rho$, there exists a $\rho^{\prime}\left(\rho<\rho^{\prime}<1\right)$ so that we have for every sequence $n_{k}$ a corresponding sequence $m_{k}$ such that $\left|a_{m_{k}}\right|>\left(\rho^{\prime}\right)^{m_{k}}$ and $m_{k}>c n_{k}$ for some $c>0$. If we choose $r$ so that $1 / \rho^{\prime}<r<1 / \rho$ we have

$$
M_{n_{k}}(r)>\left(\rho^{\prime} r\right)^{m_{k}}>\left(\rho^{\prime} r\right)^{c n_{k}}
$$

for some $c>0$ where $\rho^{\prime} r>1$.

On the other hand if we choose $r^{\prime}$ so that $r<r^{\prime}<1 / \rho$ and if (3) holds, there exists a sequence $n_{k}$ so that $f_{n_{k}}(x)$ has only $o(n)$ roots within the circle of radius $r^{\prime}$. We write

$$
f_{n}(x)=g_{n}(x) h_{n}(x) \quad\left(n=n_{k}\right)
$$

where

$$
g_{n}(x)=\Pi\left(1-\frac{x}{y_{i}}\right), \quad h_{n}(x)=\Pi\left(1-\frac{x}{z_{i}}\right)
$$

and $y_{i}$ are the roots inside, $z_{i}$ the roots outside the circle of radius $r^{\prime}$. Therefore 
the degree of $h_{n}(x)$ is $o(n)$. There clearly exists an $l<1$ such that

$$
f_{n}(x) \neq 0 \quad \text { for }|x| \leqq l
$$

(since $f(0)=1$ ). Thus

$$
\lim \left(f_{n}(x)\right)^{1 / n}=1 \quad \text { for }|x| \leqq l
$$

where that determination of $f_{n}(x)$ is taken which is 1 when $x=0$. Also

$$
\lim \left(h_{n}(x)\right)^{1 / n}=1 \quad \text { for }|x|<l .
$$

Therefore from (30) and (31)

$$
\lim \left(g_{n}(x)\right)^{1 / n}=1 \quad \text { for }|x|<l .
$$

We have

$$
g_{n}(x) \leqq \Pi\left(1+\left|\frac{x}{y_{i}}\right|\right) \leqq\left(1+\frac{|x|}{r^{\prime}}\right)^{n} \leqq 2^{n} \quad \text { for }|x| \leqq r^{\prime} .
$$

Thus by Vitali's theorem (by (32) and (33))

$$
\lim \left(g_{n}(x)\right)^{1 / n}=1 \quad \text { for }|x| \leqq r<r^{\prime} .
$$

From

$$
\max _{|x| \leq r} h_{n}(x) \leqq\left(1+\frac{r}{l}\right)^{o(n)}
$$

we obtain from (34)

$$
\left|f_{n}(x)\right|=\left|g_{n}(x)\right|\left|h_{n}(x)\right|<(1+\delta)^{2 n} \quad \text { for }|x| \leqq r
$$

for arbitrarily small $\delta>0$ and sufficiently large $k$. Therefore we have

$$
\lim \sup \left(\left|M_{n_{k}}(r)\right|\right)^{1 / n_{k}} \leqq 1,
$$

which contradicts (29). This completes the proof of Theorem II.

Let $\sum_{k=0}^{\infty} a_{k} x^{k}\left(a_{0}=1\right)$ be a power series of radius of convergence 1 which has Ostrowski gaps. Let $f_{n_{k}}(x)=1+\cdots+a_{n_{k}} x^{n_{k}}$ and $\lim \left|a_{n_{k}}\right|^{1 / n_{k}}=1 / l$. Bourion $\left({ }^{2}\right)$ remarks that every boundary point of the region of overconvergence of $f_{n_{k}}(x)$ has a distance from the origin which is less than a constant depending on $l$. In fact by using the concept of transfinite diameter $\left({ }^{8}\right)$ it is easy to see that this constant is less than $4 l$. We are going to show that this constant is greater than $l$.

Let $T_{n}(x)$ be the $n$th Tschebicheff polynomial belonging to the interval $(0,4)$. It is well known that the maximum of $T_{n}(x)$ in $(0,4)$ equals 2 . We de-

(3) For the definition and properties of the transfinite diameter see M. Fekete, Math. Zeit. vol. 17 (1923) pp. 228-249. The result we need is that the transfinite diameter of an interval of length $l$ is $l / 4$. 
note by $A_{n}$ the largest coefficient (in absolute value) of $T_{n}(x)$. It is easy to see that $\lim \left|A_{n}\right|^{1 / n}<4$. Let $n_{i}$ tend to infinity sufficiently fast and consider the power series

$$
f(x)=\sum_{i=1}^{\infty} x^{m_{i}} \frac{T_{n_{i}}(x)}{A_{n_{i}}}, \quad \quad m_{i}=m_{i-1}+n_{i-1}+1
$$

Put

$$
f_{n_{k}+m_{k}}(x)=\sum_{i=1}^{k} x^{m_{i}} \frac{T_{n_{i}}(x)}{A_{n_{i}}} .
$$

It is easy to see that if the $n_{i}$ tend to infinity sufficiently fast the circle of convergence of $f(x)$ is $1, \lim \left(1 / A_{n_{k}}\right)^{1 /\left(n_{k}+m_{k}\right)}>1 / 4$ and every interior point of $(-1,4)$ is in the region of overconvergence of $f_{n_{k}+m_{k}}(x)$. This completes the proof.

Let us denote by $\phi(l)$ the maximum distance of a boundary point of the region of overconvergence from the origin. We have

$$
l<\phi(l)<4 l \text {. }
$$

The question of the exact value of $\phi(l)$ remains open.

Added in proof. P. Turán recently pointed out that Lemma I is a consequence of the following theorem of Van Vleck (see, for example, Dieudonné, La theorie analytique des polynomes d'une variable a coefficients quelconques (Mémorial des Sciences Mathématiques, vol. 93), Paris, Gauthier-Villars, 1939). Let $h(z)=b_{0}+\cdots+b_{n} z^{n}$ and $\alpha$ be the unique positive root of

$$
C_{n-1, p-1}\left|b_{0}\right|+C_{n-2, p-2}\left|b_{1}\right| x+\cdots+C_{n-p, 0}\left|b_{p-1}\right| x^{p-1}-\left|b_{n}\right| x^{n}=0 \text {. }
$$

Then $h(z)$ has at least $p$ roots in $|z| \leqq \alpha$.

More precisely, Turán obtains the following result: Let $\rho>\rho^{\prime}>1$, $0<\theta<1 / 10$, and

$$
\theta \log \frac{20}{\theta}<\frac{9}{20} \log \frac{\rho}{\rho^{\prime}}
$$

and $n$ sufficiently large. Then if $f(z)=1+\cdots+a_{n} z^{n},\left|a_{\nu}\right|<\rho^{-\nu}$ for $m<\nu<n$, $f(z)$ has for $|z|>\rho^{\prime}$ at least $\theta(n-m)$ roots.

Turán obtains this result by a simple computation, by applying Van Vleck's theorem with $p=[\theta(n-m)]+1$ to $z^{n} f(1 / z)$.

University of Pennsylvania and

Swarthmore College 